\title{
Problems and Life Effects Experienced by Tinnitus Research Study Volunteers: An Exploratory Study Using the ICF Classification
}

DOI: $10.3766 /$ jaaa.17094

\author{
Vinaya Manchaiah* $* \ddagger \S$ \\ Eldré W. Beukes $\uparrow$ \\ Sarah Granberg\#\| \\ Naresh Durisala** \\ David M. Baguley $\uparrow \nmid \dagger+\ddagger$ \\ Peter M. Allen $₫ \S \S$ \\ Gerhard Andersson $\llbracket \uparrow \# \#$
}

\begin{abstract}
Background: Tinnitus is one of the most distressing hearing-related symptoms. It is often associated with a range of physiological and psychological complications, such as depression, anxiety, and insomnia. Hence, approaching tinnitus from a biopsychological perspective may be more appropriate than from purely a biomedical model.

Objective: The present study was aimed at determining the relationship between tinnitus and the problems and
life effects experienced by UK-based tinnitus research study volunteers. Open-ended questions were used. Re-
sponses were classified using the International Classification of Functioning, Disability and Health (ICF) frame-
work to understand the impact of tinnitus in a multidimensional manner using a biopsychosocial perspective.
\end{abstract}

Research Design: A cross-sectional survey design was used.

Study Sample: Study sample included a sample of 240 adults with tinnitus who were interested in undertaking an Internet-based intervention for tinnitus.

Data Collection and Analysis: The data were collated using two open-ended questions. The first focused on problems related to having tinnitus and the second to life effects as a result of tinnitus. Responses were analyzed using a simplified content analysis approach to link concepts to ICF categories in accordance with established linking rules. A Wilcoxon signed-rank test was performed to compare the number of responses between the two questions.

Results: There were 764 responses related to problems identified, 797 responses associated with life effects due to tinnitus, and 37 responses that did not fit into any ICF category. No significant differences were observed in the number of responses between the two questions. In addition, no significant association between the number of responses reported and demographic variables was found. Most of the problems and life effects experienced by tinnitus sufferers were related to body function, followed by activity limitations, and participation restrictions. Only a few responses were related to environmental and personal factors. The most

*Department of Speech and Hearing Sciences, Lamar University, Beaumont, TX; †Linnaeus Centre Head, Swedish Institute for Disability Research, Department of Behavioral Science and Learning, Linköping University, Linköping, Sweden; †Audiology India, Mysore, Karnataka, India; §Department of Speech and Hearing, School of Allied Health Sciences, Manipal University, Manipal, Karnataka, India; $₫$ Department of Vision and Hearing Sciences, Anglia Ruskin University, Cambridge, United Kingdom; \#The Swedish Institute for Dișability Research (SIDR), School of Health Sciences, Örebro University, Orebro, Sweden; \|Audiological Research Center, Örebro University Hospital, Örebro, Sweden; ** GN Hearing Pte Ltd, CT Hub, Singapore; + National Institute for Health Research - Nottingham Hearing Biomedical Research Unit, Division of Clinical Neuroscience, School of Medicine, University of Nottingham, Nottingham, United Kingdom; + +tology and Hearing Group, Division of Clinical Neurosciences, Faculty of Medicine, University of Nottingham, Nottingham, United Kingdom; §§Vision and Eye Research Unit, Anglia Ruskin University, Cambridge, United Kingdom; $\uparrow \uparrow D$ Department of Behavioral Sciences and Learning, Linköping University, Linköping, Sweden; \#\#Department of Clinical Neuroscience, Division of Psychiatry, Karolinska Institute, Stockholm, Sweden

Corresponding author: Vinaya Manchaiah, Department of Speech and Hearing Sciences, Lamar University, Beaumont, TX 77710; Email: vinaya.manchaiah@lamar.edu

This article presents independent research funded by the National Institute for Health Research. The views expressed are those of the authors and not necessarily those of the National Health Service, the National Institute for Health Research, or the Department of Health. 
frequent responses related to body function involved "emotional functions" (b152), "sleep functions" (b134), "hearing functions" (b230), "sustaining attention" (b1400), and "energy level" (b1300). For activity limitations and participation restrictions they were "communicating with—receiving—spoken messages" (d310), "socialization" (d9205), "handling stress and other psychological demands" (d240), and "recreation and leisure" (d920). The most frequently occurring responses related to environmental factors were "sound intensity" (e2500), "sound quality" (e2501), and "general products and technology for communication" (e1250). "Coping styles" was the most frequently occurring personal factor.

Conclusions: The study highlights the use of open-ended questions in gathering useful information about the impact of tinnitus. The responses coded to ICF show that tinnitus impacts many domains, not only particularly body function, but also activity limitations and participation restrictions. The results demonstrate the heterogeneous nature of the impact of tinnitus on people affected.

Key Words: activity limitations, body function, ICF, open-ended questions, participation restrictions, tinnitus

Abbreviations: ICF = International Classification of Functioning, Disability and Health; LEQ = life effects question; $\mathrm{PQ}=$ problem question; $\mathrm{THI}=$ Tinnitus Handicap Inventory

\section{INTRODUCTION}

$\mathrm{T}$ innitus is one of the most distressing hearingrelated symptoms (Cima et al, 2011). It is defined as the perception of sound in the absence of an external sound source (Baguley et al, 2013). It is often associated with a range of physiological and psychological complications, such as depression, anxiety, and insomnia (Langguth, 2011). Tinnitus has a high prevalence rate, estimated to be $10-15 \%$ of the adult population across the globe (Davis and Rafie, 2000; Khedr et al, 2010; Michikawa et al, 2010; Shargorodsky et al, 2010). Although many of those with tinnitus are able to habituate, others find the impact on activities of daily living devastating (Moroe and Khoza-Shangase, 2014). Tinnitus experiences are, therefore, varied and may depend on many factors unrelated to the tinnitus sound itself (Andersson, 2002). To date there is no "cure" to permanently abolish tinnitus, and standardized assessment and effective treatment methods remain elusive (Baguley et al, 2013).

Approaching tinnitus from a biopsychological perspective, which focuses on the interaction of biologic factors such as genetics, psychological factors, including mood and personality, and social factors such as cultural and socioeconomic, may be more appropriate than from purely a biomedical model (Engel, 1980). To help conceptualize the impact of health conditions and disability, the World Health Organization created a comprehensive framework based on a biopsychosocial approach, namely the International Classification of Functioning, Disability and Health (ICF; World Health Organization, 2001). This framework has been applied extensively to understand the impact of various health conditions including hearing loss (Danermark et al, 2010; Granberg, Dahlström, et al, 2014; Granberg, Möller, et al, 2014; Granberg, Pronk, et al, 2014; Granberg, Swanepoel, et al, 2014; Stephens et al, $2001 ; 2003)$. The ICF classification contains approximately 1,500 categories related to health, divided into the components body structures (s); body functions $(b)$; activities and participation $(d)$; environmental factors $(e)$; and personal factors $(p f)$. The personal factors currently contain no fixed categories; however, a broad categorization has been proposed by some audiology researchers (Stephens, 2002; Stephens and Danermark, 2005). The ICF acknowledges a noncausal relation between the components. The term "functioning" denotes the positive aspects and "disability," the negative aspects of the interaction between an individual (with a health condition) and the individual's contextual factors (i.e., environmental and personal factors). Disability is an umbrella term for impairments, activity limitations, and participation restrictions.

Tinnitus is considered as an aspect of body function according to the ICF, that is, "b2400-ringing in ears or tinnitus" (World Health Organization, 2001). However, its impact involves various elements related to activity limitations and participation restrictions, or other body functions. A study explored the impact of tinnitus using the ICF perspective (Ramkumar and Rangasayee, 2010). Their results suggest that in those experiencing tinnitus, various body functions (e.g., emotional function and sleep function) are more severely affected than aspects of activity limitations and participation restrictions. A standardized questionnaire, namely the Tinnitus Handicap Inventory (THI; Newman et al, 1996), was mapped to ICF categories to conduct the analysis. The construct of this questionnaire is predominantly related to body function (about 70\%). Hence, this measure may not have explored all the dimensions of tinnitus impact in a comprehensive manner. Building on this evidence by investigating whether tinnitus has an impact on other dimensions is required. Using open-ended questions could provide more flexibility for participants to highlight wider issues that are important to them.

Some researchers suggest that open-ended questionnaires can be clinically useful in exploring wider aspects related to clinical conditions and provide clinically more useful information than some of the structured questionnaires (Stephens et al, 2001; Stephens and 
Pyykko, 2011). Tyler and Baker (1983) explored difficulties caused by tinnitus using a single open-ended question in 72 people with tinnitus who were members of a self-help group. A small positive correlation was found between recent tinnitus onset and the number of difficulties identified. The main difficulties identified fell into four categories, which included (a) interference with hearing; (b) effect on lifestyle (mainly sleep); (c) health effects (drug use, pain, and headache); and (d) emotional effects (depression, distraction, and confusion). However, other than Ramkumar and Rangasayee (2010) who used a structured questionnaire, we are not aware of any studies that have explored tinnitus impact in detail using the ICF.

The aim of the present study was to map (link) the responses from open-ended questions regarding the impact of tinnitus using the biopsychosocial perspective of the ICF framework.

\section{METHOD}

\section{Ethical Considerations}

Ethical approval was obtained from the Faculty of Science and Technology Research Ethics Panel of Anglia Ruskin University (FST/FREP/14/478), Cambridge, United Kingdom.

\section{Study Design and Participants Recruitment}

A cross-sectional survey design was used. The data were collected during the recruitment of participants for a clinical trial investigating the feasibility and effectiveness of Internet-based cognitive behavioral therapy in the United Kingdom (Beukes et al, 2015; Beukes, Allen, et al, 2017; Beukes, Baguley, et al, 2017).

Adults (18 yr and above) living in the United Kingdom and experiencing tinnitus for a minimum period of 3 months were invited to participate in the study. Recruitment was UK-wide in various formats, including online (e.g., the National Health Service Choices), Twitter (British Tinnitus Association), Facebook forums (e.g., Action on Hearing loss), newspapers, and magazines (e.g., Mature Times), support groups (e.g., tinnitus and thyroid) and from professionals (general practitioners clinic and audiologists). Those interested in taking part provided informed consent before participation.

\section{Data Collection}

The data collection was carried out online during the registration for the study, before undertaking the intervention. The data collection was multimodal and included both structured and open-ended questions. Two hundred and forty chronic tinnitus patients completed the questionnaires.
The following structured questionnaires were administered

- A demographic questionnaire to obtain information related to age, gender, tinnitus, and hearing difficulties.

- The Tinnitus Functional Index (Meikle et al, 2012) to determine the level of tinnitus severity.

- The Insomnia Severity Index (Bastien et al, 2001) was used to determine the presence of insomnia as sleep difficulties are prevalent among those with tinnitus (Crönlein et al, 2016).

- The Generalized Anxiety Disorder (Spitzer et al, 2006) was used to quantify the level of anxiety as the prevalence of anxiety is high in persons with severe tinnitus (Pinto et al, 2014).

In addition, to allow participants to fully express any effects of tinnitus, two open-ended questions were asked. These questions were related to the impact tinnitus may have in terms of problems faced and the life effects related to tinnitus: (a) A problem question (PQ): Make a list of difficulties, which you have as a result of your tinnitus. Write down as many as you can think of; (b) a life effects question (LEQ): Make a list of the effects your tinnitus has on your life. Write down as many as you can think of. For consistency, the questions used were based on those used by previous studies related to hearing loss (Barcham and Stephens, 1980; Stephens et al, 2001; Durisala et al, 2017). Responses were required for both questions. If there were no problems or effects, participants could indicate this by saying "none."

\section{Data Analysis}

\section{Quantitative Analysis}

The Statistical Package for Social Sciences version 23.0 was used for quantitative analysis.

Descriptive statistics regarding the means and standard deviations were obtained. For the open-ended questions, the number of individual responses for $\mathrm{PQ}$ and LEQ and total responses were determined. To determine if there were significant differences between the number of responses for each question (in all ICF domains), the Wilcoxon signed-rank test was performed as the data were not normally distributed. To determine the relationship between the number of problems and life effects mentioned and demographic and clinical variables, the Spearman's rho correlation coefficient was calculated as some of the data were nonparametric. For all analyses, a two-tailed significance level of $<0.05$ was considered statistically significant. However, Bonferroni corrected significance levels (i.e., $0.05 / 5$ comparisons $=0.01$ ) were used to interpret the significance levels in multiple comparisons. 


\section{Qualitative Analysis and ICF Mapping}

In the first instance, reported problems were coded into small and meaningful units using a simplified qualitative content analysis (Graneheim and Lundman, 2004), for example, "This is something I have to cope with every minute of every day and night, no matter where I am or what I am doing" ("coping or regulating emotions," "daily routine"). In many cases, however, single words or short phrases were used requiring no real content analysis. In those cases, a single word or phrase was considered as the meaningful concept. In the next stage, these meaningful units were categorized into four main areas as defined by the ICF (i.e., functional impairment, activity limitation and participation restrictions, environmental factors, or personal factors) and ICF codes were assigned. All responses were translated ("linked") to the ICF based on established linking rules (Cieza et al, 2005; Granberg, Möller, et al, 2014). The objective of the linking process is to translate concepts found in the participants' responses into the most appropriate ICF categories. The ICF does not provide detailed classification for personal factors. Hence, a classification proposed by audiology researchers was used to classify personal factors (Stephens, 2002; Stephens and Danermark, 2005). Information that was not possible to assign to any ICF category was labeled $n d$ (not definable), $n c$ (not covered by ICF), or $h c$ (health condition). Two researchers (N.D. and S.G.) independently reviewed the responses and conducted the linking to increase the reliability of the linking procedure. If there was a disagreement (i.e., this occurred in approximately $20 \%$ of the original coding), it was discussed and if an agreement could still not be reached (i.e., approximately 5\% of the coding after discussion), a third researcher (V.M.) was consulted.

\section{RESULTS}

\section{Study Sample}

There were 240 adults (137 males and 103 females) with an average tinnitus severity indicating severe levels of tinnitus (see Table 1). The mean tinnitus duration was more than $10 \mathrm{yr}$, and more than $80 \%$ of participants had some degree of a self-reported hearing disability.

\section{Number of Responses}

There were 1,599 responses to the two open-ended questions with 765 from the PQ and 797 from the LEQ questionnaire. There were 22 responses (15 for $\mathrm{PQ}$ and 7 from LEQ) that could not be categorized using the ICF classification and were hence coded as $n c$. There were also 15 responses ( 6 for PQ and 9 from LEQ) that
Table 1. Demographic Information of Participants

\begin{tabular}{lc}
\hline Category & $\begin{array}{c}\text { Number/Mean } \\
(\mathrm{n}=240)\end{array}$ \\
\hline Gender & $137(57.1 \%)$ \\
$\quad$ Male (n/\%) & $103(42.9 \%)$ \\
$\quad$ Female (n/\%) & $56.95(\mathrm{SD}=12.51)$ \\
Age (years) & $11.52(\mathrm{SD}=11.88)$ \\
Tinnitus duration (years) & $55.16(\mathrm{SD}=21.86)$ \\
Tinnitus severity (TFI) & $12.01(\mathrm{SD}=6.81)$ \\
Insomnia (Insomnia Severity Index) & $6.93(\mathrm{SD}=5.66)$ \\
Anxiety level (Generalized & \\
$\quad$ Anxiety Disorder-7) & \\
Self-reported difficulty hearing & \\
$\quad$ None & $47(19.6 \%)$ \\
$\quad$ Slight & $131(54.6 \%)$ \\
$\quad$ Moderate & $51(21.3 \%)$ \\
$\quad$ Great & $11(4.5 \%)$ \\
\hline
\end{tabular}

Note: SD = standard deviation.

were related to a different health condition that were coded as $h c$.

The number of meaningful responses ranged from 0 to 10 , while most respondents provided 2-3 meaningful responses for both questions, as shown in Figure 1. The mean number of responses per participant was 3.2 and 3.3 for $\mathrm{PQ}$ and LEQ, respectively. No significant differences were observed in the number of responses between the two questions in all the ICF domains as seen in Table 2.

The Spearman's rho correlation showed that the strength of the relationship between the number of problems listed for the $\mathrm{PQ}$ and the LEQ was weak for the demographical variables of age, gender, and tinnitus duration (see Table 3). The strength of the relationship was also weak for tinnitus severity for the $\mathrm{PQ}$ and marginal for the number of life effects. The correlation was positive for tinnitus severity and gender, negative for age, and linear for gender. Because of the nonlinear nature of the relationships, the correlations for these measures are low even though a significant proportion of the data were accounted for by the relationship between the two variables.

\section{Impairment of Body Function}

Impairment of body function was the most frequently listed problem and life effect associated with tinnitus. There were 731 responses with a similar distribution for both questions, with 350 responses from the $\mathrm{PQ}$ and 381 from the LEQ, as shown in Table 4. The most frequently occurring category was "emotional functions" (b152) with 202 responses. Other frequently occurring categories included: "sleep functions" (b134), "hearing functions" (b230), "sustaining attention" (b1400), and "energy level" (b1300). 

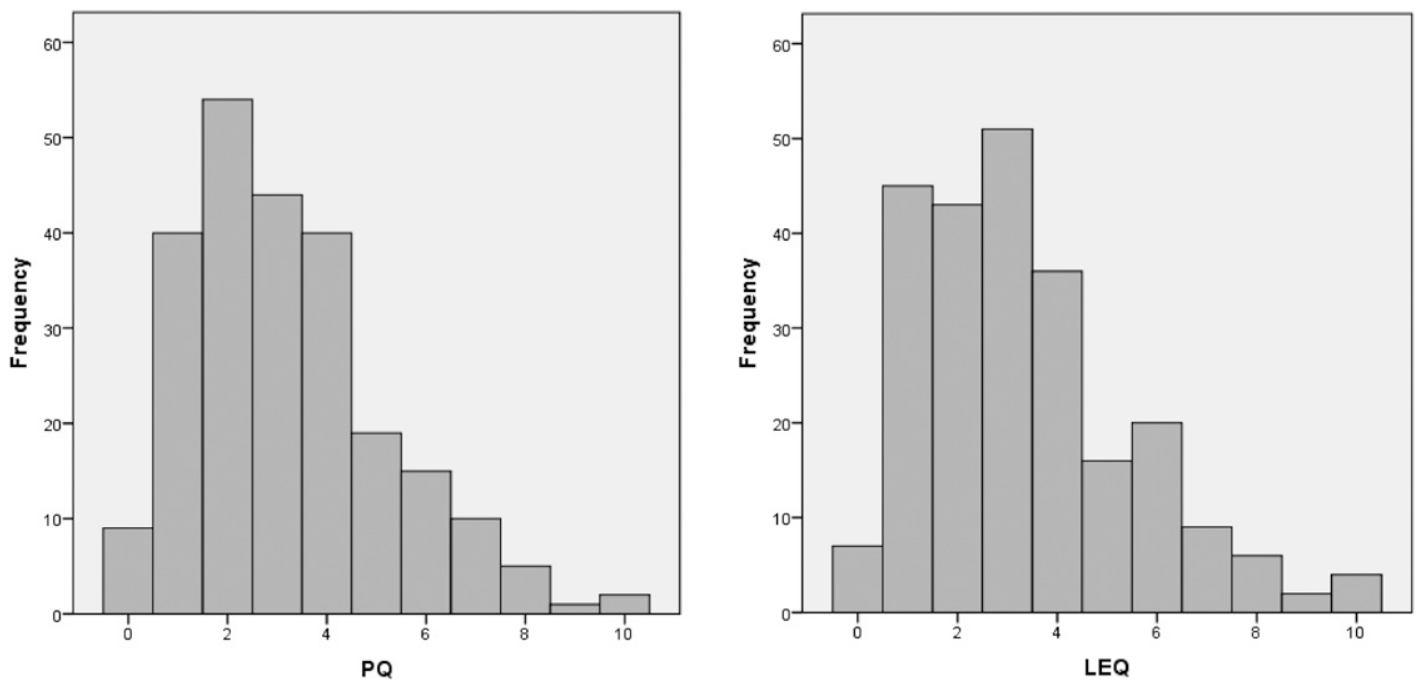

Figure 1. Number of problems listed in PQ and LEQ.

\section{Activity Limitations and Participation Restrictions}

Activity limitations and participation restrictions were impacted second most frequently with 518 responses as shown in Table 5. There were an equal number of responses to the PQ (i.e., 258) and LEQ (i.e., 260). The most frequently occurring category was "communicating with-receiving-spoken messages" (d310), which had 83 responses. This was followed by "socialization" (d9205), "handling stress and other psychological demands" (d240), and "recreation and leisure" (d920).

\section{Environmental Factors}

Environmental factors included both inanimate and animate factors in the world that may influence an individual. Table 6 presents all the ICF categories related to environmental factors in participants. The most frequently occurring categories included "sound intensity" (e2500), "sound quality" (e2501), and "general products and technology for communication" (e1250).

\section{Personal Factors}

Personal factors was the ICF category with the least mention from this sample of research volunteers experiencing tinnitus as seen in Table 7. These factors are specific to an individual and influence the problems and life experience reported/experienced. "Coping styles" was the single most frequently occurring personal factor related to tinnitus.

\section{Overall Impact of Tinnitus}

A summary of the ICF functions most frequently impacted by tinnitus is shown in Figure 2. "Emotional functions" and "sleep functions" were the functions most affected.

\section{DISCUSSION}

$T$ his study explored problems and life effects experienced by 240 tinnitus research volunteers using an open-ended questioning approach. Responses were coded using the ICF classification system. There were 1,561 responses in total. Most of the individuals provided 2-3 meaningful responses to each question. This indicates the multidimensional nature of tinnitus and that it impacts various domains. It also indicates that including an open-ended approach has great value in obtaining information related to the impact of tinnitus. There was only a weak or very weak relationship between the number of problem and/or life effects

Table 2. Number of Responses in Each of the ICF Domains Listed in the Two Questions

\begin{tabular}{|c|c|c|c|c|}
\hline Category & PQ Mean (SD) & LEQ Mean (SD) & Wilcoxon Z & Sig. \\
\hline All responses & $3.2(2.0)$ & $3.33(2.1)$ & -0.99 & 0.32 \\
\hline Functional impairment (body function) & $1.46(1.1)$ & $1.59(1.2)$ & -1.38 & 0.17 \\
\hline Activity limitations and Participation restriction & $1.08(1.2)$ & $1.09(1.2)$ & -0.15 & 0.98 \\
\hline Environmental factors & $0.50(0.8)$ & $0.56(0.8)$ & -1.17 & 0.24 \\
\hline Personal factors & $0.15(0.4)$ & $0.09(0.3)$ & -1.94 & 0.05 \\
\hline
\end{tabular}

Note: SD = standard deviation. 
Studying Tinnitus Using the ICF/Manchaiah et al

Table 3. Relationship between Number of Responses to Problem and Life Effects Question and the Demographic Variables Such as Tinnitus Severity, Age, Duration, Gender

\begin{tabular}{|c|c|c|c|c|c|}
\hline \multirow[b]{2}{*}{ Question } & \multirow[b]{2}{*}{ Comparison } & \multicolumn{4}{|c|}{ Spearman's rho Correlation } \\
\hline & & $\overline{r_{s}}$ & $p$ & $R^{2}$ & $\%$ of variability \\
\hline \multirow[t]{4}{*}{$\overline{\mathrm{PQ}(\mathrm{all})}$} & TFI & 0.21 & 0.002 & 0.042 & 4.20 \\
\hline & Age & 0.13 & 0.042 & 0.017 & 1.70 \\
\hline & Duration & 0.08 & 0.225 & 0.002 & 0.20 \\
\hline & Gender & 0.17 & 0.006 & 0.031 & 3.10 \\
\hline \multirow[t]{4}{*}{ LEQ (all) } & $\mathrm{TFI}$ & 0.33 & 0.000 & 0.106 & 10.60 \\
\hline & Age & 0.05 & 0.472 & 0.002 & 0.20 \\
\hline & Duration & 0.10 & 0.117 & 0.006 & 0.60 \\
\hline & Gender & 0.11 & 0.103 & 0.011 & 1.10 \\
\hline
\end{tabular}

mentioned and the demographic variables such as age, gender, and tinnitus duration and clinical variable tinnitus severity.

\section{Functioning and Disability}

Body functions was the area most frequently affected. "Emotional functions" (b152) was the domain with the most responses overall at 202 responses. This may be related to effects such as anxiety, stress, depression, and negative thinking patterns often associated with tinnitus (Langguth, 2011). This finding accentuates the need for appropriate assessment measures that fully investigate emotional functioning in those with tinnitus. From the mapping done of the THI distribution on ICF domains by Ramkumar and Rangasayee

Table 4. Impairments of Body Functions

\begin{tabular}{|c|c|c|c|c|}
\hline$\overline{\text { Function }}$ & ICF Code & $P Q(n=350)$ & $\mathrm{LEQ}(\mathrm{n}=381)$ & Total $(n=731)$ \\
\hline Temperament and personality function & b126 & 6 & 5 & 11 \\
\hline Extraversion & b1260 & 1 & 0 & 1 \\
\hline Psychic stability & b1263 & 8 & 7 & 15 \\
\hline Optimism & b1265 & 2 & 1 & 3 \\
\hline Confidence & b1266 & 5 & 3 & 8 \\
\hline Energy and drive functions & b130 & 3 & 3 & 6 \\
\hline Energy level & b1300 & 23 & 20 & 43 \\
\hline Motivation & b1301 & 1 & 2 & 3 \\
\hline Appetite & b1302 & 3 & 1 & 4 \\
\hline Sleep function & b134 & 71 & 94 & 165 \\
\hline Amount of sleep & b1340 & 0 & 1 & 1 \\
\hline Onset of sleep & b1341 & 5 & 13 & 18 \\
\hline Maintenance of sleep & b1342 & 1 & 1 & 2 \\
\hline Attention function & b140 & 12 & 22 & 34 \\
\hline Sustaining attention & b1400 & 38 & 45 & 83 \\
\hline Memory functions & b144 & 0 & 1 & 1 \\
\hline Emotional functions & b152 & 108 & 94 & 202 \\
\hline Thought functions & b160 & 15 & 8 & 23 \\
\hline Hearing functions & b230 & 36 & 37 & 74 \\
\hline Sound discrimination & b2301 & 1 & 3 & 4 \\
\hline Localization of sound source & b2302 & 0 & 3 & 3 \\
\hline Speech discrimination & b2304 & 0 & 1 & 1 \\
\hline Vestibular function of balance & b2351 & 1 & 1 & 2 \\
\hline Sensation associated with hearing and vestibular function & b240 & 2 & 0 & 2 \\
\hline Dizziness & b2401 & 1 & 1 & 2 \\
\hline Nausea associated with dizziness and vertigo & b2403 & 1 & 1 & 2 \\
\hline Aural pressure & b2405 & 0 & 1 & 1 \\
\hline Sensation of pain & b280 & 2 & 1 & 3 \\
\hline Pain in head and neck & b28010 & 1 & 9 & 10 \\
\hline Sexual functions & b640 & 1 & 1 & 2 \\
\hline Muscle power function & b730 & 1 & 1 & 2 \\
\hline Muscle endurance function & b740 & 1 & 0 & 1 \\
\hline
\end{tabular}


Table 5. Activity Limitations and Participation Restrictions

\begin{tabular}{|c|c|c|c|c|}
\hline Function & ICF Code & $P Q(n=258)$ & $\operatorname{LEQ}(n=260)$ & Total $(n=518)$ \\
\hline Watching & $\mathrm{d} 110$ & 1 & 1 & 2 \\
\hline Listening & d115 & 19 & 17 & 36 \\
\hline Copying & d130 & 0 & 1 & 1 \\
\hline Acquiring skills & d155 & 1 & 0 & 1 \\
\hline Focusing attention & d160 & 1 & 1 & 2 \\
\hline Thinking & d163 & 3 & 1 & 4 \\
\hline Reading & d166 & 8 & 18 & 26 \\
\hline Making decisions & d177 & 1 & 0 & 1 \\
\hline Undertaking a single task & $\mathrm{d} 210$ & 1 & 0 & 1 \\
\hline Understanding multiple tasks & d220 & 0 & 3 & 3 \\
\hline Carrying out daily routine & d230 & 2 & 0 & 2 \\
\hline Handling stress and other psychological demands & d240 & 26 & 22 & 48 \\
\hline Communicating with—receiving—spoken messages & d310 & 35 & 48 & 83 \\
\hline Speaking & d330 & 1 & 0 & 1 \\
\hline Conversation & d350 & 14 & 23 & 37 \\
\hline Conversing with one person & d3503 & 1 & 1 & 2 \\
\hline Conversing with many people & d3504 & 6 & 3 & 9 \\
\hline Using communication devices & d360 & 1 & 0 & 1 \\
\hline Using telecommunication devices & d3600 & 4 & 12 & 16 \\
\hline Using communication techniques & d3602 & 6 & 3 & 9 \\
\hline Walking & $\mathrm{d} 450$ & 0 & 1 & 1 \\
\hline Moving around in different locations & $\mathrm{d} 460$ & 1 & 2 & 3 \\
\hline Using transportation & d470 & 2 & 5 & 7 \\
\hline Using human powered vehicles & $\mathrm{d} 4700$ & 1 & 0 & 1 \\
\hline Using public motorized transportation & $\mathrm{d} 4702$ & 1 & 0 & 1 \\
\hline Driving & $\mathrm{d} 475$ & 1 & 3 & 4 \\
\hline Drinking & d560 & 1 & 0 & 1 \\
\hline Shopping & d6200 & 0 & 4 & 4 \\
\hline Basic interpersonal interactions & $\mathrm{d} 710$ & 2 & 0 & 2 \\
\hline Complex interpersonal interactions & d720 & 0 & 1 & 1 \\
\hline Interacting according to social rules & d7203 & 2 & 0 & 2 \\
\hline Informal social relationships & d750 & 2 & 0 & 2 \\
\hline Informal relationships with friends & d7500 & 0 & 1 & 1 \\
\hline Family relationships & $\mathrm{d} 760$ & 8 & 5 & 13 \\
\hline Parent-child relationships & d7600 & 0 & 1 & 1 \\
\hline Child parent relationships & d7601 & 1 & 2 & 3 \\
\hline Extended family relationships & d7603 & 1 & 0 & 1 \\
\hline Intimate relationships & $\mathrm{d} 770$ & 1 & 0 & 1 \\
\hline Spousal relationships & d7701 & 3 & 0 & 3 \\
\hline Interpersonal interactions and relations, unspecified & d799 & 1 & 1 & 2 \\
\hline Remunerative employment & d850 & 17 & 18 & 35 \\
\hline Community life & d910 & 4 & 0 & 4 \\
\hline Recreation and leisure & d920 & 25 & 19 & 44 \\
\hline Sports & d9201 & 3 & 1 & 4 \\
\hline Arts and culture & d9202 & 20 & 16 & 36 \\
\hline Hobbies & d9204 & 1 & 0 & 1 \\
\hline Socialization & d9205 & 27 & 23 & 50 \\
\hline Religion and spirituality & d930 & 2 & 3 & 5 \\
\hline
\end{tabular}

(2010), mental functions accounted for 18 of the 25 THI items. Mapping of these domains on other commonly used tinnitus assessment measures is required. Assessing emotional functioning in those with tinnitus on assessment instruments designed to identify anxiety and depression is, therefore, of value. This finding also highlights the importance of addressing emotional function- ing during tinnitus interventions. Psychologically based interventions such as cognitive behavioral therapy are structured to address these issues (Andersson, 2002).

"Sleep functions" (b134) was the domain with the second most overall responses with 165 responses. This highlights the strong link between tinnitus and quality 


\begin{tabular}{|c|c|c|c|c|}
\hline Function & ICF Code & $P Q(n=120)$ & LEQ $(n=134)$ & Total $(n=254)$ \\
\hline$\overline{\text { Food }}$ & e1100 & 1 & 3 & 4 \\
\hline Drugs & e1101 & 1 & 2 & 3 \\
\hline Products and technology for personal use in daily living & e115 & 5 & 4 & 9 \\
\hline General products and technology for personal use in daily living & e1150 & 1 & 2 & 3 \\
\hline Assistive products and technology for personal use in daily living & e1151 & 6 & 1 & 7 \\
\hline Products and technology for communication & e125 & 3 & 7 & 10 \\
\hline General products and technology for communication & e1250 & 21 & 24 & 45 \\
\hline Assistive products and technology for communication & e1251 & 4 & 4 & 8 \\
\hline Products and technology for education & e130 & 1 & 0 & 1 \\
\hline Assistive products and technology for culture, recreation and sport & e1401 & 1 & 0 & 1 \\
\hline $\begin{array}{l}\text { Design, construction and building products, and technology for } \\
\text { entering and exiting buildings for public use }\end{array}$ & e150 & 4 & 2 & 6 \\
\hline Financial assets & e1650 & 0 & 1 & 1 \\
\hline Climate & e225 & 0 & 1 & 1 \\
\hline Time related changes & e245 & 2 & 0 & 2 \\
\hline Sound & e250 & 5 & 12 & 17 \\
\hline Sound intensity & e2500 & 30 & 26 & 56 \\
\hline Sound quality & e2501 & 21 & 34 & 55 \\
\hline Immediate family & e310 & 2 & 1 & 3 \\
\hline Friends & e320 & 1 & 0 & 1 \\
\hline Individual attitudes of immediate family members & e410 & 3 & 4 & 7 \\
\hline Individual attitudes of friends & e420 & 0 & 1 & 1 \\
\hline Societal attitudes & e460 & 1 & 0 & 1 \\
\hline Attitudes, others specified & e498 & 2 & 1 & 3 \\
\hline Attitudes unspecified & e499 & 4 & 2 & 6 \\
\hline Media services & e5600 & 1 & 2 & 3 \\
\hline
\end{tabular}

of sleep. Assessing this domain using appropriate outcome measures is important to identify where problems lie and subsequently to design a management plan. Addressing sleep difficulties, when present, should form an integral part of tinnitus management services. Also, "hearing functions" (b230) had 74 responses and was the fourth most frequently occurring category. As hearing loss is one of the greatest risk factors for developing tinnitus, this is perhaps to be expected (Shargorodsky et al, 2010); although, this may also be indicative of the impact of tinnitus upon auditory processing, leading to increased listening effort (Degeest et al, 2017). Accessibility to the newest technology in the hearing aid field, which aids both hearing loss and tinnitus problems, is important. Patient

Table 7. Personal Factors

\begin{tabular}{lccc}
\hline Function & $\begin{array}{c}\mathrm{PQ} \\
(\mathrm{n}=36)\end{array}$ & $\begin{array}{c}\text { LEQ } \\
(\mathrm{n}=22)\end{array}$ & $\begin{array}{c}\text { Total } \\
(\mathrm{n}=58)\end{array}$ \\
\hline Lifestyle & 2 & 1 & 3 \\
Habits & 1 & - & 1 \\
Coping styles & 27 & 17 & 44 \\
Profession & 1 & 2 & 3 \\
$\begin{array}{l}\text { Past and present } \\
\quad \text { experience }\end{array}$ & 2 & - & 2 \\
$\begin{array}{l}\text { Overall behavior patterns } \\
\text { and characteristics }\end{array}$ & 3 & 2 & 5 \\
\hline
\end{tabular}

education on the potential of these devices is important as the stigma of wearing hearing aids remains in many cultures (David and Werner, 2016). The fourth most frequently impacted area was "sustaining attention" (b1400). A systematic review by Tegg-Quinn et al (2016) concluded that tinnitus impairs cognitive function because of its impact on executive control of attention. Hearing loss, anxiety, and depression may further exacerbate these effects. Exploring these effects in those with tinnitus is important so that they can be appropriately addressed.

In terms of activity limitations and participation restrictions, "Communicating with—receiving—spoken messages" (d310) was most frequently reported with 83 responses followed by "socialization" (d9205) with 50 responses. These findings are related to those of Degeest et al (2017) who explored listening effort in normal-hearing participants with chronic tinnitus. Listening effort increased across various listening conditions when compared with those without tinnitus. This will also have an impact on socialization. Moreover, those with tinnitus often avoid social situations due to fear of these having a negative impact on their tinnitus (Hesser and Andersson, 2009). This, in turn, impacts socialization and also "recreation and leisure" (d920), which was also a domain frequently affected. Another domain featured in this category was "handling 


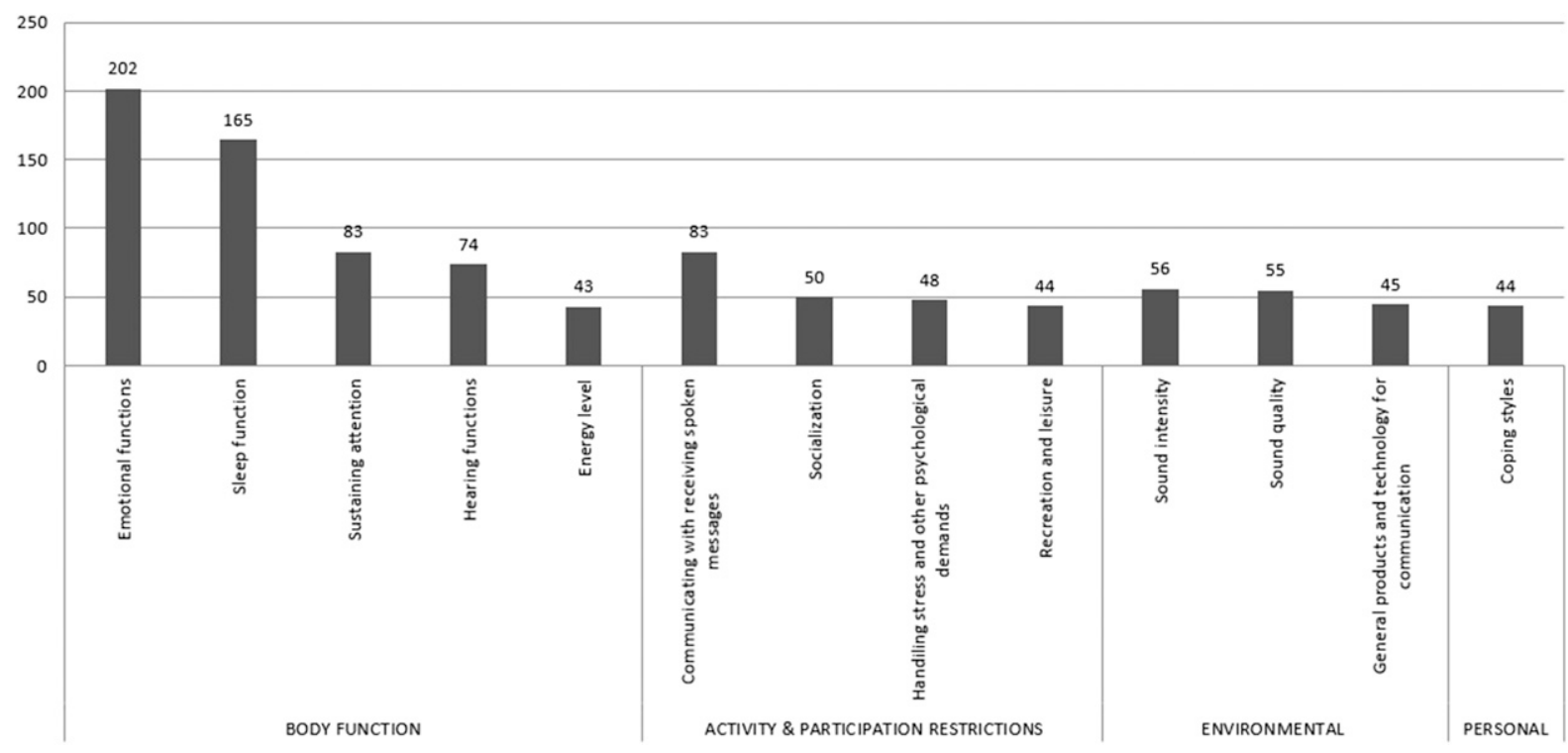

Figure 2. The most frequently reported factors impacted due to tinnitus.

stress and other psychological demands" (d240). In a large epidemiological population study in Sweden, stress was found to be an important risk factor for severe tinnitus (Baigi et al, 2011). Tinnitus management needs to include assessment of these domains (i.e., communication, recreation and leisure, handling stress and other psychological demands), and should provide those with tinnitus effective coping mechanisms to ensure that activities and participation restrictions are less problematic.

The present study results are in line with a previous study by Ramkumar and Rangasayee (2010), suggesting that the component "body functions" is the main element that is affected in tinnitus sufferers with a mean score being almost twice that of activity and participation restrictions. However, in the present study, the domain of activity limitations and participation restrictions was also found to be affected in people with tinnitus, which is in contrast to the previous study. There were numerous methodological differences between the studies as the previous study used a smaller sample size $(\mathrm{n}=21)$ and used a structured questionnaire. The average tinnitus duration in the previous study was $2.05 \mathrm{yr}$, which is much lower than the average duration of $11.52 \mathrm{yr}$ in the present study. The impact of tinnitus in the acute and prolonged stages of the condition may, therefore, differ. In addition, the present study included people with tinnitus both with and without hearing loss, whereas Ramkumar and Rangasayee's study included people with tinnitus without hearing loss. Patients with tinnitus with and without hearing loss have different characteristics (Hallberg and Erlandsson, 1993), which may have resulted in the difference in findings.

\section{Contextual Factors}

Contextual factor domains were featured less in this study. "Sound intensity" (e2500) and "sound quality" (e2501) were reported 56 and 55 times, respectively. Those with tinnitus often comment on the overpowering nature of tinnitus. Various objective measures focus on understanding the frequency, intensity, and masking level required to mask the tinnitus. These measures have, however, been found to be unrelated to the degree of distress caused by tinnitus (Erlandsson et al, 1992). Hearing aids are often provided to try to assist in reducing the intensity of tinnitus as well as to improve hearing. Frustrations when hearing aids do not provide enough benefit were mentioned in the domain "general products and technologies for communication" (e1250). Although hearing aids can help in reducing tinnitus distress, evidence to support or refute their use is still required (Hoare et al, 2014).

"Coping styles" was the only personal factor that was discussed frequently in the present study participants. Hallberg et al (1992) observed coping strategies used by males with noise induced-hearing loss having tinnitus $(\mathrm{n}=89)$. Coping strategies are more likely to include drinking alcohol and wishful thinking in people with severe tinnitus, along with strategies common to less severe groups matched on other relevant variables. In the present study, the main coping style was ignoring the tinnitus or becoming habituated to it.

\section{Study Implications}

The use of the biopsychosocial perspective has aided comprehensive understanding of the impact of tinnitus. 
The implications are of value at several different levels. Assessment methods should focus on the functions most frequently affected and can inform the development of future outcome measures. There is a strong movement toward applying appropriate outcome measures, especially in the context of clinical trials (Williamson et al, 2012). The Tinnitus Research Initiative also has prioritized creating appropriate outcome measures for tinnitus (Langguth et al, 2007), and concerns about this issue continue to be raised (Londero and Hall, 2017). A systematic review indicated that the outcome domains most frequently used in the clinical trials of tinnitus relate to the tinnitus percept, the impact of tinnitus, other co-occurring complains, health-related quality of life, and body structures and functions (Hall et al, 2016). Results of the present study could be used to ensure future outcome measures that target the domains most frequently found to be problematic in those with tinnitus. Moreover, the use of open-ended questions may be used as add-on questions in addition to structured questionnaires designed to gather individual specific information from tinnitus sufferers (Stephens and Pyykko, 2011).

Considering the average tinnitus duration of this sample was more than $10 \mathrm{yr}$, it indicates that the impact of tinnitus may continue regardless of tinnitus duration. Those with tinnitus may benefit from more accessible tinnitus interventions that can help address these problem areas. The results should also be used to guide the development and planning of future tinnitus interventions so that they focus on the areas identified. Reducing the impairments of body functions (e.g., sleep functions, attention functions, and emotional functions), and also reducing the activity limitations and participation restrictions (e.g., handling stress and other psychological demands and socialization) should be the key focus of audiological management.

\section{Strengths and Limitations of the Study}

The study used open-ended questions in a crosssectional design administered online. Online administration of questionnaires may have an advantage. For example, it is easier to read the responses of participants when compared with the paper and pencil method. Open-ended questions have the advantage of being useful in exploring wider aspects related to tinnitus than can be obtained from structured questionnaires (Stephens et al, 2001; Stephens and Pyykko, 2011). For the purposes of this study, this approach is perhaps more ecologically valid as it provides the opportunity for participants to decide the problems experienced rather than imposing the responses and ideas on them in structured questionnaires. Open-ended questions, however, have drawbacks such as making it difficult for some to remember or come up with responses, and can lead to a lower response rate (about 40-50\%; Manchaiah, Baguley, et al, 2015). The response rate to the questions in this study was high as a response was required from all participants to each question presented. A further strength of the study was the experience in linking ICF codes of multiple authors of this study. However, the contextual factors (i.e., environmental and personal factors) can act as both facilitators and a hindrance to health and disability. Hence, they can be coded as positive, negative, or neutral influencing factors (Manchaiah, Möller, et al, 2015), which was not done in this study because of limited contextual information in the questionnaire responses.

Participants in this study were recruited from a UKbased population that had expressed interest in being involved in a trial of an Internet-based treatment program for tinnitus. This may have introduced certain biases, which may limit the generalizability of the findings to a wider population of people with tinnitus. Volunteering for the study may imply that other treatment approaches have not been effective. It may be that having the time to consider being involved in a research study could be associated with being unemployed or retired. The motivation for being involved may be associated with particularly severe tinnitus.

\section{CONCLUSIONS}

$T$ he study explored problems and life effects experienced by people with tinnitus. No significant differences were observed in the number of responses for different elements between the two questions (i.e., a PQ and an LEQ). Most of the problems and life effects experienced by tinnitus sufferers were related to body function followed by activity limitations and participation restrictions. However, limited responses were related to environmental and personal factors. The most frequently occurring responses related to body function were "emotional functions" (b152), "sleep functions" (b134), "hearing functions" (b230), "sustaining attention" (b1400), and "energy level" (b1300). The most frequently occurring responses related to activity limitations and participation restrictions included "communicating with—receiving—spoken messages" (d310), "socialization" (d9205), "handling stress and other psychological demands" (d240) and "recreation and leisure" (d920). The most frequently occurring responses related to environmen7tal factors were "sound intensity" (e2500) and "sound quality" (e2501). Also, the most frequently occurring personal factor was related to "coping styles." The results suggested that the open-ended questions were found to be useful in gathering useful information about problems and life effects experienced by tinnitus sufferers. The responses coded to the ICF show that tinnitus sufferers have a wide range of issues, although some issues, mainly in body functions, seem to stand 
out and are more common. The results demonstrate the heterogeneous nature of the impact of tinnitus on people affected and has implications toward tinnitus management. Future research should focus on ensuring tinnitus assessment methods and interventions for tinnitus sufferers as these are problem areas. Future ICF coding for tinnitus could combine closed and open-ended questions as this provides a more comprehensive assessment and is advised both in research and clinical practice.

\section{REFERENCES}

Andersson G. (2002) Psychological aspects of tinnitus and the application of cognitive-behavioral therapy. Clin Psychol Rev 22(7): 977-990

Baguley DM, Andersson G, McKenna L. (2013) Tinnitus: A Multidisciplinary Approach. 2nd ed. Chichester, UK: Wiley-Blackwell.

Baigi A, Oden A, Almlid-Larsen V, Barrenäs ML, Holgers KM. (2011) Tinnitus in the general population with a focus on noise and stress: a public health study. Ear Hear 32(6):787-789.

Barcham LJ, Stephens SDG. (1980) The use of an open-ended problems questionnaire in auditory rehabilitation. $\mathrm{Br} J$ Audiol 14(2):49-54.

Bastien CH, Vallières A, Morin CM. (2001) Validation of the Insomnia Severity Index as an outcome measure for insomnia research. Sleep Med 2:297-307.

Beukes EW, Allen PM, Manchaiah V, Baguley DM, Andersson G. (2017) Internet-based intervention for tinnitus: outcome of a singlegroup open trial. J Am Acad Audiol 28(4):340-351.

Beukes E, Baguley DM, Allen PM, Manchaiah V, Andersson G. (2017) Guided internet-based cognitive behaviour therapy for adults with tinnitus in the United Kingdom: a randomised controlled trial. Ear Hear In Press.

Beukes EW, Manchaiah V, Allen PM, Baguley DM, Andersson G. (2015) Internet-based cognitive behavioural therapy for adults with tinnitus in the UK: study protocol for a randomised controlled trial. BMJ Open 5(9):e008241.

Cieza A, Geyh S, Chatterji S, Kostanjsek N, Ustün B, Stucki G. (2005) ICF linking rules: an update based on lessons learned. $J$ Rehabil Med 37(4):212-218.

Cima RF, Vlaeyen JW, Maes IH, Joore MA, Anteunis LJ. (2011) Tinnitus interferes with daily life activities: a psychometric examination of the tinnitus disability index. Ear Hear 32(5):623-633.

Crönlein T, Langguth B, Pregler M, Kreuzer PM, Wetter TC, Schecklmann M. (2016) Insomnia in patients with chronic tinnitus: cognitive and emotional distress as moderator variables. J Psychosom Res 83:65-68.

Danermark B, Cieza A, Gangé J-P, Gimigliano F, Granberg S, Hickson L, Kramer SE, McPherson B, Möller C, Russo I, Strömgren JP, Stucki G, Swanepoel D. (2010) International classification of functioning, disability, and health core sets for hearing loss: a discussion paper and invitation. Int J Audiol 49(4): $256-262$

Davis A, Rafie EA. (2000) Epidemiology of tinnitus. In: Tyler RS, ed. Tinnitus Handbook. San Diego, CA: Singular Thomson Learning, 1-23.

David D, Werner P. (2016) Stigma regarding hearing loss and hearing aids: a scoping review. Stigma Health 1(2):59-71.
Degeest S, Keppler H, Corthals P. (2017) The effect of tinnitus on listening effort in normal-hearing young adults: a preliminary study. J Speech Lang Hear Res 60(4):1036-1045.

Durisala N, Manchaiah V, Granberg S, Möller K. (2017) Determination and classification of the problems experienced by adults with single-sided deafness using ICF classification: an exploratory study using 26 participants. Clin Otolaryngol 42(3): $748-752$.

Engel GL. (1980) The clinical application of the biopsychosocial model. Am J Psychiatry 137(5):535-544.

Erlandsson SL, Hallberg LRM, Axelsson A. (1992) Psychological and audiological correlates of perceived tinnitus severity. Audiology $31: 168-179$.

Granberg S, Dahlström J, Möller C, Kähäri K, Danermark B. (2014) The ICF core sets for hearing loss-researcher perspective. Part I: systematic review of outcome measures identified in audiological research. Int $J$ Audiol 53(2):65-76.

Granberg S, Möller K, Skagerstrand A, Möller C, Danermark B. (2014) The ICF core sets for hearing loss: researcher perspective, Part II: linking outcome measures to the International Classification of Functioning, Disability and Health (ICF). Int $J$ Audiol 53(2):77-87.

Granberg S, Pronk M, Swanepoel de W, Kramer SE, Hagsten H, Hjaldahl J, Möller C, Danermark B. (2014) The ICF core sets for hearing loss project: functioning and disability from the patient perspective. Int J Audiol 53(11):777-786.

Granberg S, Swanepoel W, Englund U, Möller C, Danermark B. (2014) The ICF core sets for hearing loss project: international expert survey on functioning and disability of adults with hearing loss using the International Classification of Functioning, Disability, and Health (ICF). Int J Audiol 53(8):497-506.

Graneheim UH, Lundman B. (2004) Qualitative content analysis in nursing research: concepts, procedures and measures to achieve trustworthiness. Nurse Educ Today 24(2):105-112.

Hall DA, Haider H, Szczepek AJ, et al. (2016) Systematic review of outcome domains and instruments used in clinical trials of tinnitus treatments in adults. Trials 17(1):270.

Hallberg LR-M, Erlandsson SI. (1993) Tinnitus characteristics in tinnitus complainers and noncomplainers. $B r$ J Audiol 27(1):19-27.

Hallberg LR-M, Erlandsson SI, Carlsson SG. (1992) Coping strategies used by middle-aged males with noise-induced hearing loss with and without tinnitus. Psychol Health 7:273-288.

Hesser H, Andersson G. (2009) The role of anxiety sensitivity and behavioral avoidance in tinnitus disability. Int $J$ Audiol 48(5): 295-299.

Hoare DJ, Edmondson-Jones M, Sereda M, Akeroyd MA, Hall D. (2014) Amplification with hearing aids for patients with tinnitus and co-existing hearing loss. Cochrane Database Syst Rev (1): CD010151.

Khedr EM, Ahmed MA, Shawky OA, Mohamed ES, El Attar GS, Mohammad KA. (2010) Epidemiological study of chronic tinnitus in Assiut, Egypt. Neuroepidemiology 35(1):45-52.

Langguth B. (2011) A review of tinnitus symptoms beyond 'ringing in the ears': a call to action. Curr Med Res Opin 27(8): $1635-1643$.

Langguth B, et al. (2007) Consensus for tinnitus patient assessment and treatment outcome measurement: Tinnitus Research 
Initiative meeting, Regensburg, July 2006. Prog Brain Res 166: $525-536$.

Londero A, Hall DA. (2017) Call for an evidence-based consensus on outcome reporting in Tinnitus Intervention Studies. Front Med (Lausanne) 4:42.

Manchaiah V, Baguley DM, Pyykkö I, Kentala E, Levo H. (2015) Positive experiences associated with acquired hearing loss, Ménière's disease, and tinnitus: a review. Int J Audiol 54(1): $1-10$.

Manchaiah V, Möller K, Pyykkö I, Durisala N. (2015) Capturing positive experiences of a health condition such as hearing loss and balance disorders when using the ICF framework. Hear Balance Commun 13(3):134-136.

Meikle MB, et al. (2012) The tinnitus functional index: development of a new clinical measure for chronic, intrusive tinnitus. Ear Hear 33(2):153-176.

Michikawa T, Nishiwaki Y, Kikuchi Y, Saito H, Mizutari K, Okamoto M, Takebayashi T. (2010) Prevalence and factors associated with tinnitus: a community-based study of Japanese Elders. J Epidemiol 20(4):271-276.

Moroe NF, Khoza-Shangase K. (2014) The impact of tinnitus on daily activities in adult tinnitus sufferers: a pilot study. $S$ Afr $J$ Commun Disord 61(1). http://dx.doi.org/10.4102/sajcd.v61i1.65

Newman CW, Jacobson GP, Spitzer JB. (1996) Development of the tinnitus handicap inventory. Arch Otolaryngol Head Neck Surg 122(2): 143-148.

Pinto PC, Marcelos CM, Mezzasalma MA, Osterne FJV, de Melo Tavares de Lima MA, Nardi AE. (2014) Tinnitus and its association with psychiatric disorders: systematic review. J Laryngol Otol 128:660-664.

Ramkumar V, Rangasayee R. (2010) Studying tinnitus in the ICF framework. Int J Audiol 49(9):645-650.
Shargorodsky J, Curhan GC, Farwell WR. (2010) Prevalence and characteristics of tinnitus among US adults. Am J Med 123(8):711-718.

Spitzer RL, Kroenke K, Williams JB, Löwe B. (2006) A brief measure for assessing generalized anxiety disorder: the GAD-7. Arch Intern Med 166:1092-1097.

Stephens D. (2002) Audiological rehabilitation. In: Luxon L, Furman JM, Martini A, eds. Textbook of Audiological Medicine. London, UK: Martin Dunitz, 513-531.

Stephens D, Danermark B. (2005) The international classification of functioning, disability and health as a conceptual framework for the impact of genetic hearing impairment. In: Stephens D, Jones L, eds. The Impact of Genetic Hearing Impairment. London, UK: Whur, 54-67.

Stephens D, Gianopoulos I, Kerr P. (2001) Determination and classification of the problems experienced by hearing-impaired elderly people. Audiology 40(6):294-300.

Stephens DP, Pyykko I. (2011) How useful are 'add-on' questions in questionnaires? Audiol Med 9:47-48.

Stephens D, Vetter N, Lewis P. (2003) Investigating lifestyle factors affecting hearing aid candidature in the elderly. Int $J$ Audiol 42:2S33-2S38.

Tegg-Quinn S, Bennett RJ, Eikelboom RH, Baguley DM. (2016) The impact of tinnitus upon cognition in adults: a systematic review. Int $J$ Audiol 55(10):533-540.

Tyler RS, Baker LJ. (1983) Difficulties experienced by tinnitus sufferers. J Speech Hear Disord 48(2):150-154.

Williamson PR, Altman DG, Blazeby JM, Clarke M, Devane D, Gargon E, Tugwell P. (2012) Developing core outcome sets for clinical trials: issues to consider. Trials 13:132.

World Health Organization. (2001) International Classification of Functioning, Disability and Health. Geneva, Switzerland: World Health Organization. 